\title{
MHD STABILITY IN ALUMINIUM ELECTROLYSIS CELLS: FROM COMPLEX TO SIMPLE ANALYSIS
}

\author{
VALDIS BOJAREVICS ${ }^{1}$ \\ ${ }^{1}$ University of Greenwich, London, UK \\ v.bojarevics@gre.ac.uk
}

Key words: Aluminium Electrolysis, MHD stability, Shallow Water waves, Magnetohydrodynamics, Numerical Modelling, Analytical Criterion

\begin{abstract}
Numerical modelling has become a primary tool for design and optimization of commercial high amperage aluminium electrolysis cells. The design of industrial cells requires to account for a variety of their individual features: bus-bar network, cell bottom profile and side ledge, cathode bar design, ferromagnetic parts effect, operational adjustments due to the anode changes, etc. Analytical solutions in special cases provide benchmark tests and aid understanding of the basic physics. The commercial Trimet cell is analyzed using a simple theoretical, full numerical and advanced analytical problem solutions, which are compared with experimental measurements using wireless sensors to detect MHD instability onset.
\end{abstract}

\section{INTRODUCTION}

Industrial aluminium electrolytic production cells are highly optimized for magnetic field and electric current distribution in order to avoid magneto-hydrodynamic (MHD) instabilities, increase the current efficiency and to maintain a stable electrolytic process during normal operation. The mathematical modelling of the cells and the prediction of unstable behaviour has reached certain maturity since the first principles of the MHD were introduced [1-3]. The modelling has become a primary design and optimization tool of aluminium electrolysis cells. There are several commercial packages available which are being continuously developed and maintained to meet the interests of the aluminium industry. The specialised MHD code $[4,5]$ using the 3-dimensional electromagnetic fields and the extended shallow layer turbulent fluid flow model permits to simulate real commercial cells accounting for a variety of their individual features (bus-bar network, ledge, bottom profile, cathode design, ferromagnetic parts, anode change, etc.). The code was extensively tested [4,5] and fully verified against the benchmark cases [3].

However, a deeper insight to the basic mechanisms of the MHD instability has the potential 
to unravel fast and efficient ways to estimate the onset of instability using relatively simple analytical expressions [6-10]. First attempts to explain the interfacial instabilities were made by Sele [1] and Urata [7]. A more involved understanding of the physical mechanism was provided in [8-10]. The mechanism is based on the standing gravity wave modification due to the electric current redistribution. The electric current density in the electrolyte increases above the wave crests, resulting in a high-density horizontal current in the shallow liquid metal layer. In the presence of a vertical magnetic field the electromagnetic force excites another standing wave mode orthogonal to the initial perturbation. The new wave mode is coupled to the original mode, and the oscillation frequency is shifted. The frequency shift increases with the rise of the magnetic field until at a critical value, when the two wave frequencies coincide. Increasing the magnetic field further gives an exponential growth of the amplitude which indicates the onset of instability [8]. The newly derived criterion in [6] is the most comprehensive by including the effects of long gravity wave damping, the dependence on the cell geometry, the electrical conductivities and densities of the liquids, and the magnetic field distribution in the liquid layer. It is demonstrated that this criterion replicates the previously published simple cases of the stability models and the results for commercial cells where the detailed stability measurements are available $[4,5]$.

\section{MATHEMATICAL STATEMENT OF THE STABILITY CRITERION}

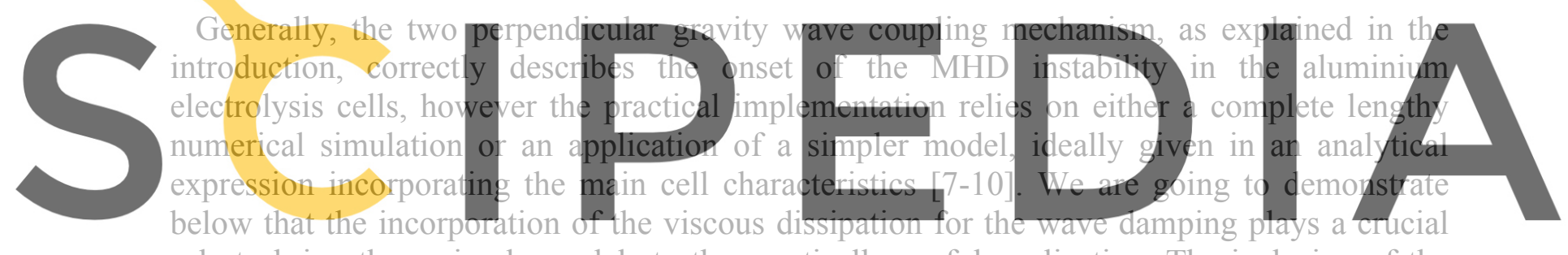

role to bring these simple models to the practically useful application. The inclusion of the

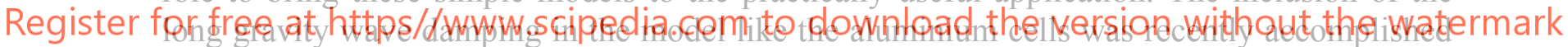

within a related problem of the MHD stability in large scale liquid metal batteries [6]. The mathematical derivation follows similar ideas as explained in [8], however including the empirical friction coefficient ' $\gamma$ ' to ensure the real shallow water wave damping times as commonly used in the geophysical or tidal flow models [11]. In this short presentation we will refer to the full derivation in [6] and will analyse the most important final result given as a relatively simple analytical criterion for the MHD instability onset in an aluminium electrolysis cell. The explicit criterion for the onset of the instability for an arbitrary two perpendicular mode interaction (in the horizontal $\mathrm{x}$ and $\mathrm{y}$ directions) can be expressed as:

$$
\gamma \leq\left(\frac{2}{\omega_{\boldsymbol{k}_{1}}^{2}+\omega_{\boldsymbol{k}_{2}}^{2}}\right)^{1 / 2}\left(\left|G_{\boldsymbol{k}_{1} \boldsymbol{k}_{2}}\right|^{2}-\left(\frac{\omega_{\boldsymbol{k}_{1}}^{2}-\omega_{\boldsymbol{k}_{2}}^{2}}{2}\right)^{2}\right)^{\frac{1}{2}} .
$$

This criterion contains the main geometrical and material parameters characterising the particular cell: the densities of the liquid metal $\rho_{1}$ and electrolyte $\rho_{2}$, the electrical conductivities $\sigma_{1}, \sigma_{2}, \sigma_{3}$ (anode graphite), the average depths of the layers $h_{1}, h_{2} h_{3}$, the horizontal dimensions of the cell $L_{x}, L_{y}$ and the empirical friction coefficient $\gamma$ (typically of 
the magnitude between 0.02 and 0.08 for the aluminium cells). The crucial function in this criterion is the magnetic field vertical component $B_{z}(x, y)$ on the surface of the liquid metal. The magnetic field distribution needs to be Fourier transformed to create the amplitudes of the corresponding modes as shown in the expression below:

$$
\widehat{B}_{k_{x}, k_{y}}=\frac{4}{L_{x} L_{y}} \int_{0}^{L_{x}} \int_{0}^{L_{y}} B_{z}(x, y) \sin \left(k_{x} x\right) \sin \left(k_{y} y\right) d x d y
$$

where the wave vector has the components

$$
\boldsymbol{k}=\left(k_{x}, k_{y}\right), \quad k_{x}=\frac{m \pi}{L_{x}}, k_{y}=\frac{n \pi}{L_{y}} .
$$

The distribution of the vertical magnetic field component $B_{z}(x, y)$ determines the wave interaction matrix needed to establish the stability of the particular cell:

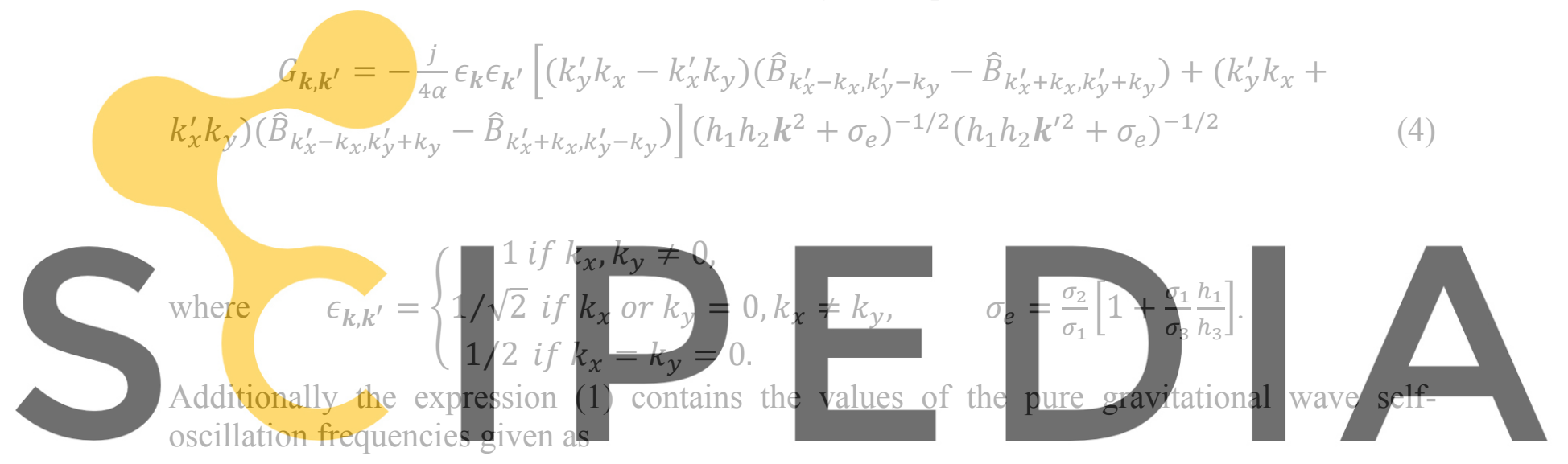

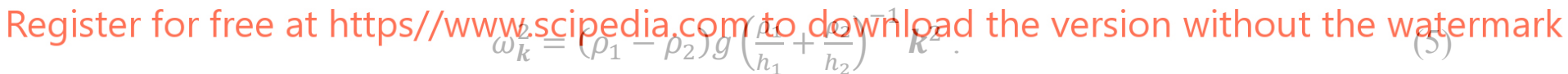

According to the criterion (1) the cell under investigation will be prone to the MHD instability when the real part of the right hand side of (1) exceeds the value of the damping coefficient $\gamma$ value. Note that the rhs contains the square root which can become a complex number when the certain modes of the magnetic field expansion (2) exceed the critical magnitude. Even in absence of the friction, when $\gamma=0$, the cell could be stable or unstable depending on the sign of the expression under the square root in (1). The full derivation of the criterion given in [6] shows that the cell will be exhibiting oscillation of the interface (also, the anode currents and voltage) at the frequency related to the original unperturbed gravity frequencies as:

$$
\Omega_{\boldsymbol{k}_{1} \boldsymbol{k}_{2}}=\sqrt{\frac{\omega_{\boldsymbol{k}_{1}}^{2}+\omega_{\boldsymbol{k}_{2}}^{2}}{2}}
$$




\section{APPLICATION EXAMPLES}

Let us illustrate the application of the criterion (1) to the previously published cases of theoretically derived MHD stability models and a practical application to a commercial cell where a detailed stability measurement case is available. We restrict our analysis to the case of the Trimet company cell where the details are given in full within the previous publication [4]. The model representation of the cell in line arrangement is shown in the Figure 1. The total line current for this cell is $180 \mathrm{kA}$, the horizontal dimensions used in the following analysis are $L_{x}=8.01 \mathrm{~m}, L_{y}=3.69 \mathrm{~m}$, the liquid metal depth is $h_{1}=0.17 \mathrm{~m}$, the electrolyte depth in the interpolar space is $h_{2}=0.045 \mathrm{~m}$.
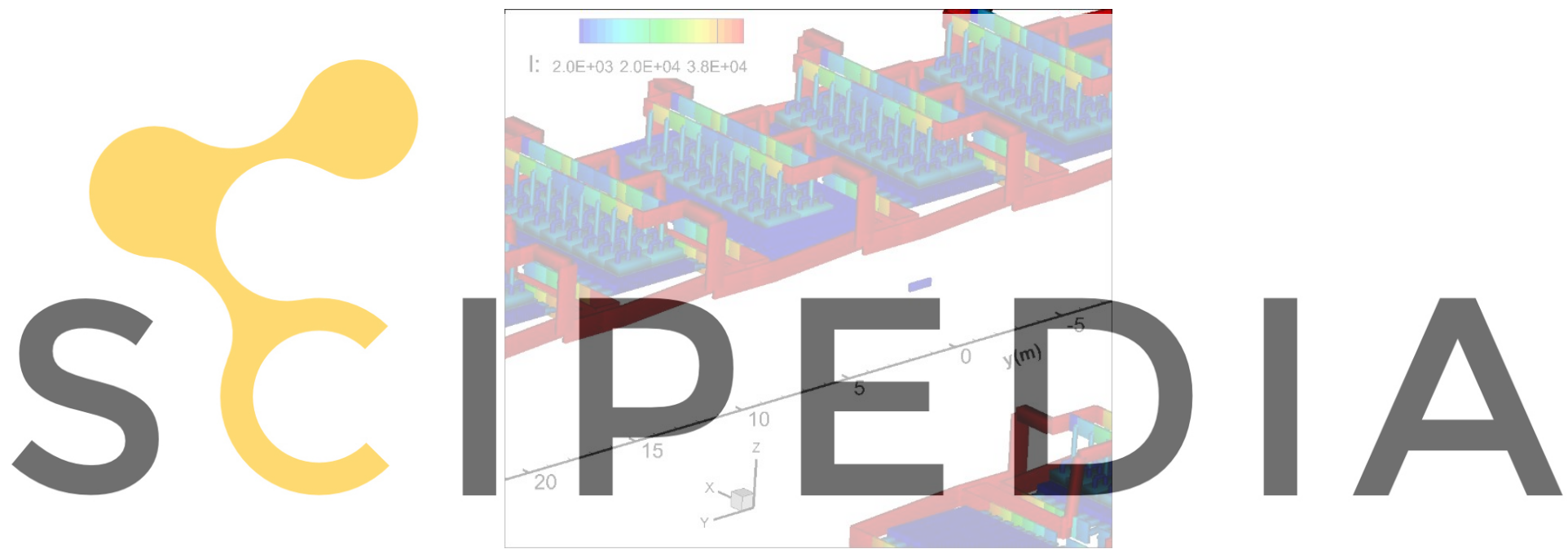

Register for free at https//www.scipedia.com to download the version without the watermark Figure 1: The model representation of the Trimet cell with the electric current distribution in the surrounding bus bar network

\subsection{The constant uniform magnetic field case}

The majority of the previously published theoretical models are restricted to the case when the wave damping is completely neglected and the magnetic field is approximated as an average constant $B_{z}$ magnitude. In the inviscid case the comparison of some model predictions for the $180 \mathrm{kA}$ Trimet cell case are shown in the Table 1. Apparently, the simple static model as presented in [7] gives a significant overestimate of the stability onset value for the magnetic field, while the other theories derived for the inviscid wave development give more or less consistent estimate of the critical magnetic field, which however is surprisingly low for this type of cell.

The numerical solution for the complete magnetic field including the supply bus bars, neighbour cells, return line and the ferromagnetic elements of the construction is shown in the Figure 2. The average value of the $B_{z}$ magnetic field is computed as $0.00147 \mathrm{~T}(1.47 \mathrm{mT})$. This computational result is supported by the measurements in operating cells [4,5]. It appears 
that inviscid fluid dynamics and the constant uniform magnetic field theories give a very low value of the critical magnetic field, which is well below the average field observed in the real cells.

Table 1 : Critical magnetic field values computed according to various theories

\begin{tabular}{|l|l|}
\hline \multicolumn{1}{|c|}{ Stability criterion source } & Critical magnetic field $\mathrm{B}_{\mathrm{z}}, \mathrm{mT}$ \\
\hline Sele [1] & 1.8 \\
\hline Sneyd, Wang [9] & 0.145 \\
\hline Davidson, Lindsay [10] & 0.130 \\
\hline Bojarevics, Romerio [8] & 0.135 \\
\hline Tucs, Bojarevics, Pericleous [6] & 0.135 \\
\hline
\end{tabular}
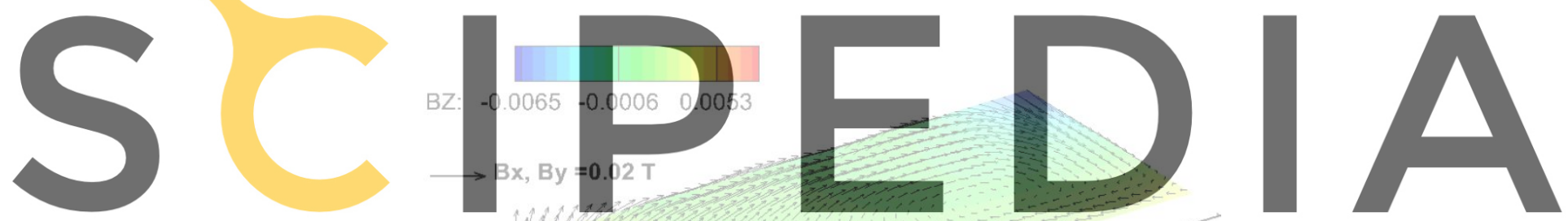

Register for free at https $4 /$ /www.scipedia.com to download the version without the watermark ros<smiles>[3H]C[CH]</smiles>

Figure 2: The computed $3 \mathrm{~d}$ magnetic field on the surface of liquid metal for the $180 \mathrm{kA}$ cell. The average value of the $B_{z}$ magnetic field is $0.00147 \mathrm{~T}(1.47 \mathrm{mT})$.

\subsection{The computed full magnetic field case and comparison to the measurements}

The magnetic field distribution shown in the Figure 2 can be used to compute the expressions required in the criterion (1) to estimate the cell stability with the wave damping friction present. The same setup can be used in the full dynamic simulation to predict the wave development over the long period of time to investigate their growth in the dependence of the interpolar distance of the electrolyte thickness. The analytical criterion predictions are summarised in the Figure 3 for the most sensitive set of combination of the interacting wave 
modes. According to the results shown in the Figure 3 the cell operation will be stable in the region above the critical stability lines, i.e., at the larger electrolyte thickness and the larger friction values. The choice of the empirical friction coefficient is determined as $\gamma=0.05$ (which also gives a good match to the horizontal velocities $\sim 0.15 \mathrm{~m} / \mathrm{s}$ ). For this value of damping the critical interpolar electrolyte depth is predicted as $0.044 \mathrm{~m}$ from the position of the top instability line in the Figure 3.
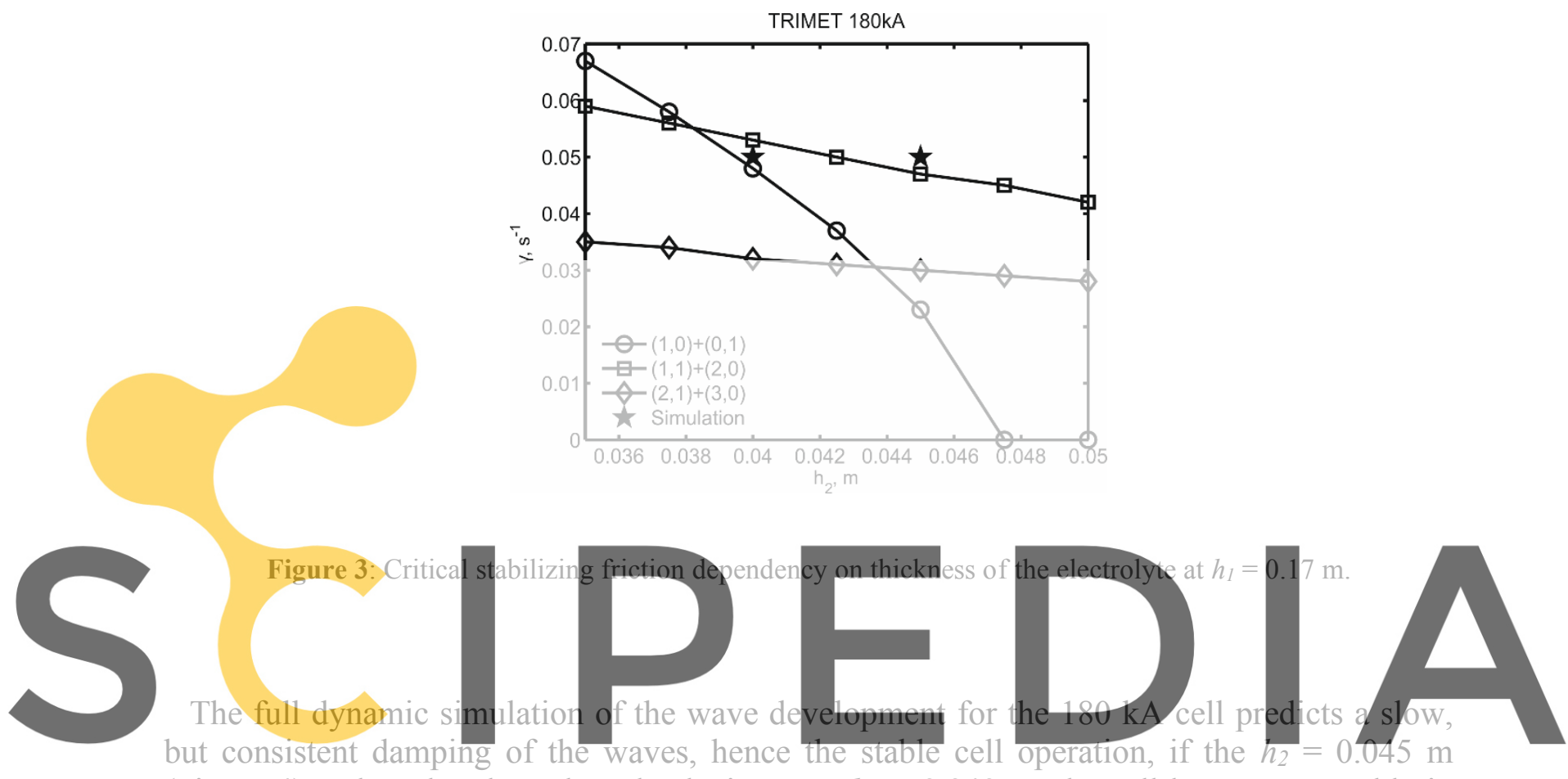
(Figure 4). When the electrolyte depth is set as $h_{2}=0.040 \mathrm{~m}$, the cell becomes unstable in

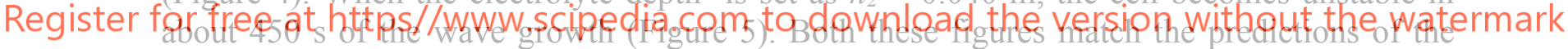

analytical criterion (1) as can be seen from the Figure 3 where the numerical test results are

shown by the star symbois. Mioreover, the oscillation frequency of $0.0254 \mathrm{~Hz}$ as observed in the experiment [4] match reasonably well the numerically computed $0.0259 \mathrm{~Hz}$. The instability onset frequency according to the analytical criterion (6) is expected to be the result of the two modes $(1 ; 1)+(2 ; 0)$ interacting. The stability limit is reached at the frequency $\mathrm{f}=$ $0.0253 \mathrm{~Hz}$, as predicted in (6).

From these results we can conclude that the stability criterion given as the analytical expression (1) provides a good initial estimate for the practical evaluation of the cell MHD stability. 

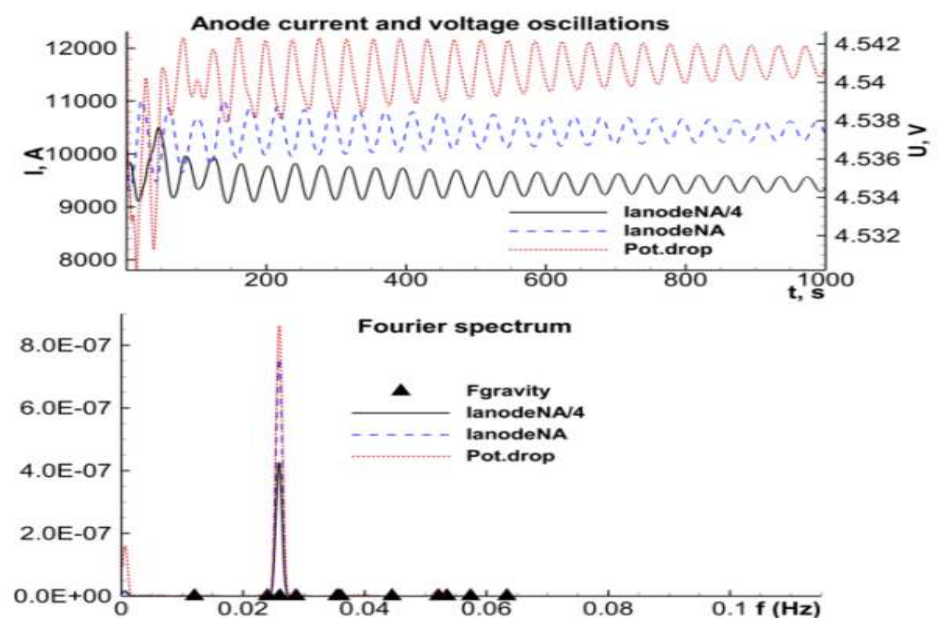

Figure 4: The electric currents in anodes and the voltage of the stable cell as computed for the $180 \mathrm{kA}$ cell at $h_{l}=0.17 \mathrm{~m}, h_{2}=0.045 \mathrm{~m}$.
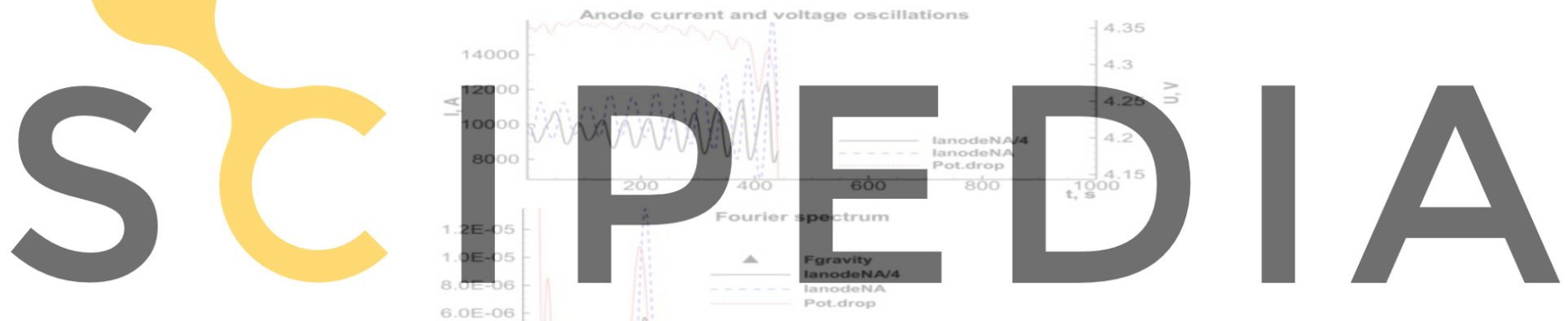

Register for free at https//WWW.scipedia.com to download the version without the watermark

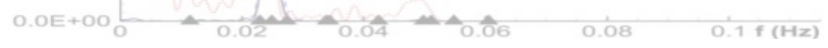

Figure 5: The electric currents in anodes and the voltage of the unstable cell as computed for the $180 \mathrm{kA}$ cell at $h_{l}=0.17 \mathrm{~m}, h_{2}=0.040 \mathrm{~m}$.

\section{SUMMARY}

- The instability in commercial Trimet cells arises when the anode-cathode distance $(\mathrm{ACD})$ is below $4.2 \mathrm{~cm}$.

- The unstable wave oscillation frequency of $0.0254 \mathrm{~Hz}$ is observed in the industrial experiment.

- $\quad$ The same stability limit is predicted by the numerical model and the new analytical criterion.

- $\quad$ The analytical criterion gives the stability limit at $\mathrm{f}=0.0253 \mathrm{~Hz}$ for the two modes $(1 ; 1)+(2 ; 0)$ interacting. 


\section{REFERENCES}

[1] Sele, T. Instabilities of the metal surface in electrolyte alumina reduction cells. Metall. Trans. (1977), 8B:613-618.

[2] Zikanov, O., Thess, A., Davidson P. A. and Ziegler D. P., A new approach to numerical simulation of the melts flows and interface instability in Hall-Heroult cells. Metall. Trans. (2000), 31B: 1541-1550.

[3] Severo, D.S., Gusberti V., Schneider A.F., Pinto E.C. and Potocnik V. Comparison of Various Methods for Modeling the Metal-Bath Interface. (2008) TMS Light Metals: 413418.

[4] Bojarevics V. and Evans J.W. Mathematical Modelling of Hall-Herault Pot Instability and Verification of Measurements of Anode Current Distribution, TMS Light Metals (2015): 783-788.

[5] Bojarevies V., Radionov E. and Tretiyakov Y. Anode bottom burnout shape and velocity field investigation in a high amperage electrolysis cell, TMS Light Metals (2018): 551556.

[6] Tucs A., Bojarevics V. and Pericleous K. Magnetohydrodynamic stability of large scale liquid metal batteries. Journal of Fluid Mechanics. (2018), 852: 453-483.

[7] Urata N. Magnetics and metal pad instability. Light Metals, (1985): 581-589.
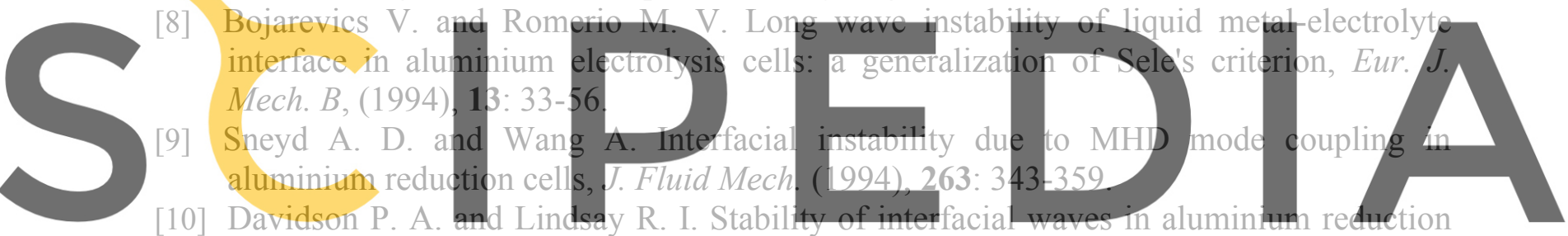
cells, J. Fluid Mech. (1998), 362: 273-295.

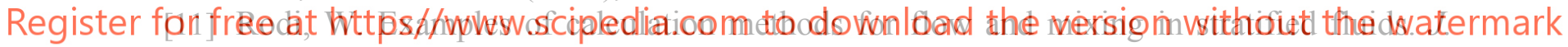
Geophys. Res. (1987), 92: 5305-5328. 